\title{
Synthesis of Some New Pyrimidines and Pyrrolo [2,3-d] Pyrimidines as Potential Antimicrobial Agents
}

\author{
Z.M. Nofal, H.H. Fahmy", E.S. Zarie and A.A. Hamdy \\ Therapeutical Chemistry Department and *Departement of \\ Natural and Microbial Products Chemistry, National Research \\ Centre, Giza, Egypt.
}

\begin{abstract}
CYCLOCONDENSATION reaction of compound 1 with oxalyl choloride in acetone and dimethyl formamide afforded pyrrolo [2,3-d] pyrimidine derivative 2 , which was reacted with $p$-amino acetophenone, p-aminobenzenesulfonamide (sulpha drugs), thiosemicarbazide, ethyl chloroacetate and benzoyl acetonitrile to give compounds 3, 4a-c, 5, 6, and 7, respectively. Condensation reaction of acetyl derivative 3 with 3, 4, 5-trimethoxybenzaldehyde afforded compound $8.2(1 \mathrm{H})$-pyridone and imino pyridine derivatives 9,10 were obtained by the reaction of compound 8 with ethyl cyanoacetate or malononitrile in presence of ammonium acetate. Moreover, chalcone derivative 8 was condensed with thiourea to give compound 11. Michel condensation reaction of 3 with ethyl cyanoacetate and 3indolcaroxaldehyde leads to the formation of the pyridine 12 in one step reaction. Bromination of compound 13 afforded 14, which was reacted with thiourea to give compound 15 . Furthermore, compound 13 was allowed to react with hydroxyl amine hydrochloride and phenyl hydrazine to give compounds 16 and 17, respectively. The antimicrobial activity of some of synthesized compounds was evaluated.
\end{abstract}

Keywords: Pyrimidine, Pyrrolo [2,3-d] pyrimidine derivatives, Condensation reaction, Cyclization and Antimicrobial activity.

Pyrimidines are well known to have a variety of biological and antimicrobial activities. Their derivatives display a wide range of pharmacological activities ${ }^{(1,2)}$. Also, it was reported that 6-phenylhydrazinouracil (I), 6-benzylaminouracil (II) and 6-anilinouracil (III) were shown to inhibit polymerase III (pol III), an enzyme known to be essential in the replicative DNA synthesis of Gr+ bacteria, of Bacillus subtilis by promoting the formation of a catalytically inactive ternary complex with DNA and the enzyme ${ }^{(3)}$.<smiles>O=c1cc(NNc2ccccc2)[nH]c(=O)[nH]1</smiles>

I<smiles>O=c1cc(NCc2ccccc2)[nH]c(=O)[nH]1</smiles>

II<smiles>Cc1ccc(Nc2cc(=O)[nH]c(=O)[nH]2)cc1C</smiles>

III

\#Corresponding author: hh_fahmy@yahoo.com 
Non classical antimetabolites, such as trimethoprim (TMP), trimetrexate (TMQ), piritrexim (PTX) and pyrimethamine (PM) are more particularly used against pathogenic microorganisms ${ }^{(4,5)}$.<smiles>COc1cc(Cc2cnc(N)nc2N)cc(OC)c1OC</smiles>

TMP<smiles>COc1cc(NCc2ccc3nc(N)nc(N)c3c2C)cc(OC)c1OC</smiles>

TMQ<smiles>COc1ccc(OC)c(Cc2cnc3nc(N)nc(N)c3c2C)c1</smiles>

PTX<smiles>CCc1nc(N)nc(N)c1-c1ccc(Cl)cc1</smiles>

$\mathrm{PM}$

On the other hand, the importance of fused pyrimidines, which are common sources for the development of new potential therapeutic agents, is well known among them the pyrrolo[2,3-d]pyrimidines are of considerable interest, they are reported to possess anti-inflammatory, anticancer and antiviral activities ${ }^{(6)}$.

Pyrrolo [2,3-d] pyrimidines are an important class of compounds, structurally and chemically related to naturally nucleoside and some antibiotics ${ }^{(7)}$, besides imidazo pyrimidine anti-mycobacterial agents ${ }^{(8)}$.

\section{Results and Discussion}

Cyclocondensation reaction of 6-amino uracil (1) with oxalyl choloride in acetone and dimethylformamide ${ }^{(9)}$ gave the pyrrolo [2,3-d] pyrimidine derivative 2 , which was condensed with $\mathrm{p}$-amino acetophenone in glacial aceticacid $^{(9)}$ to give the acetylphenyliminopyrrolo [2,3-d] pyrimidine derivative 3 .

Also, compound 2 was allowed to react with different p-aminobenzenesulfonamide derivatives (sulpha drugs), namely: sulpha pyridine, sulpha pyrimidine and sulpha isoxazole in glacial acetic $\operatorname{acid}^{(9)}$ to give the $\mathrm{N}$ - pyridine or pyrimidin or isoxazol-pyrrolo [2,3-d] pyrimidin-5-ylidene aminobenzene-sulfonamide derivatives $(4 a-c)$.

Reaction of compound 2 with thiosemicarbazide in glacial acetic acid ${ }^{(9)}$ gave 1H-pyrrolo[2,3-d]pyrimidine-2,4,5,6 (3H,7H)-tetrone 5-thiosemicarbazone (5).

Egypt. J. Chem. 54, No.5 (2011) 
Furthermore, reaction of (2) with ethyl chloroacetate in dimethyl formamide in presence of potassium carbonate anhydrous ${ }^{(10)}$ leads to the formation of Ethyl -7H-pyrrolo [2,3-d] pyrimidin-7-ylacetate (6).

On the other hand, compound 2 was reacted with phenacylcyanide in absolute ethanol and glacial acetic acid at refluxing temperature ${ }^{(11)}$ to give compound 7, it is worthy to note that IR spectrum of it showed the presence of $\mathrm{CO}-\mathrm{NH}_{2}$ instead of $\mathrm{C} \equiv \mathrm{N}$ group due to the hydrolysis of $\mathrm{C} \equiv \mathrm{N}$ to $\mathrm{CO}-\mathrm{NH}_{2}$, during the reaction (Scheme 1).

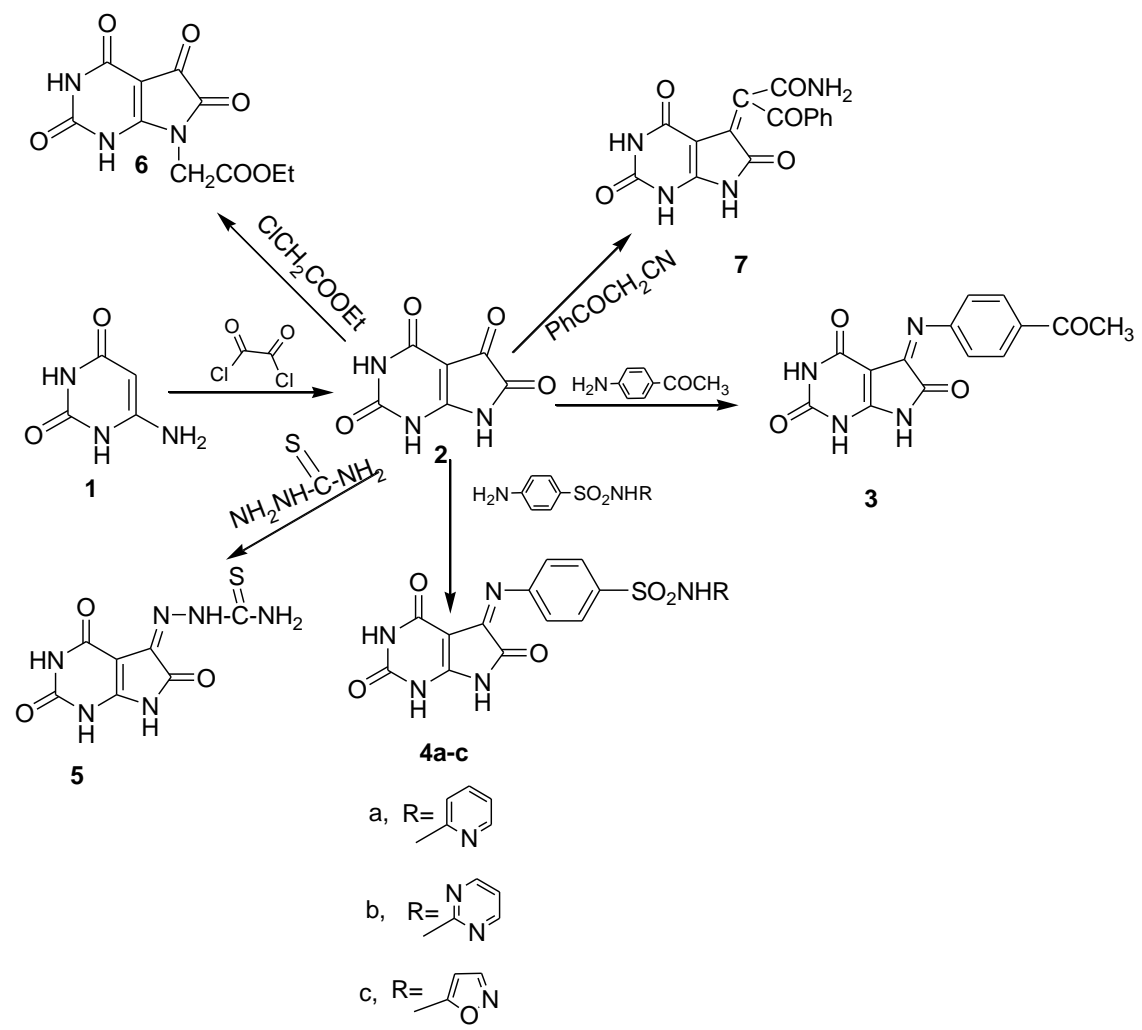

Scheme 1

Synthesis of chalcone 8 begins by Claisen-Schmidt condensation of acetyl derivative 3 with 3,4,5-trimethoxybenzaldyde in $5 \%$ ethanolic $\mathrm{NaOH}$ solution ${ }^{(12)}$.

It is well known that chalones ${ }^{(12)}$ are excellent starting materials for synthesis of $2(1 \mathrm{H})$-pyridone derivative 9 . The Aldol condensation product 8 reacts with ethyl cyanoacetate in presence of ammonium acetate to afford compound 9 . In the same manner, reaction of 8 with malononittrile under the same conditions, afforded the corresponding imino pyridine 10 . 
Also, the chalcone derivative 8 was condensed with thiourea ${ }^{(13)}$ in ethanol and a few drops of hydrochloric acid to give compound 11 .

Michael condensation of 3 with ethyl cynoacetate and 3-indolcarboxaldehyde in presence of ammonium acetate leads to the formation of the pyridone derivative 12 in one step reaction ${ }^{(12)}$ (Scheme 2$)$.

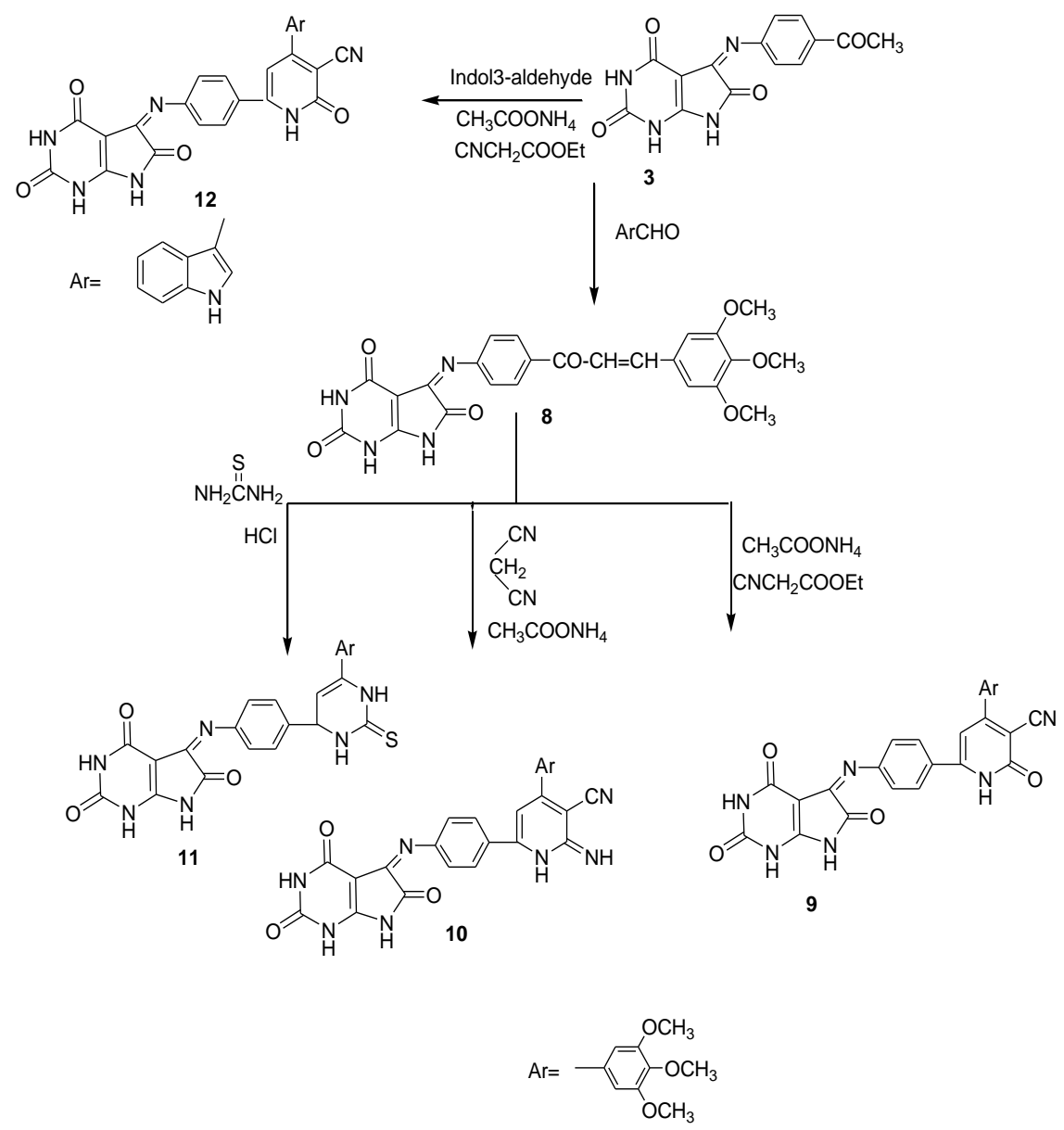

Scheme 2

Furthermore, the Schiff base ${ }^{(14)} 13$ was synthesized according to the reported method and allowed to react with bromine to form 6-amino-5-(- $\{$ [4-(bromoacetyl) phenyl] imino $\}$ methyl) pyrimidine-2,4 $(1 \mathrm{H}, 3 \mathrm{H})$-dione (14), which underwent cyclocondensation reaction with thiourea to give 6-amino-5-[4-(2-amino-1,3thiazol-4-yl) phenyl] imino $\}$ methyl) pyrimidine-2,4(1H,3H)-dione (15).

Egypt. J. Chem. 54, No.5 (2011) 
Furthermore, the Schiff base 13 was allowed to react with hydroxyl amine hydrochloride and phenyl hydrazine to give compounds 16 and 17 , respectively (Scheme 3).
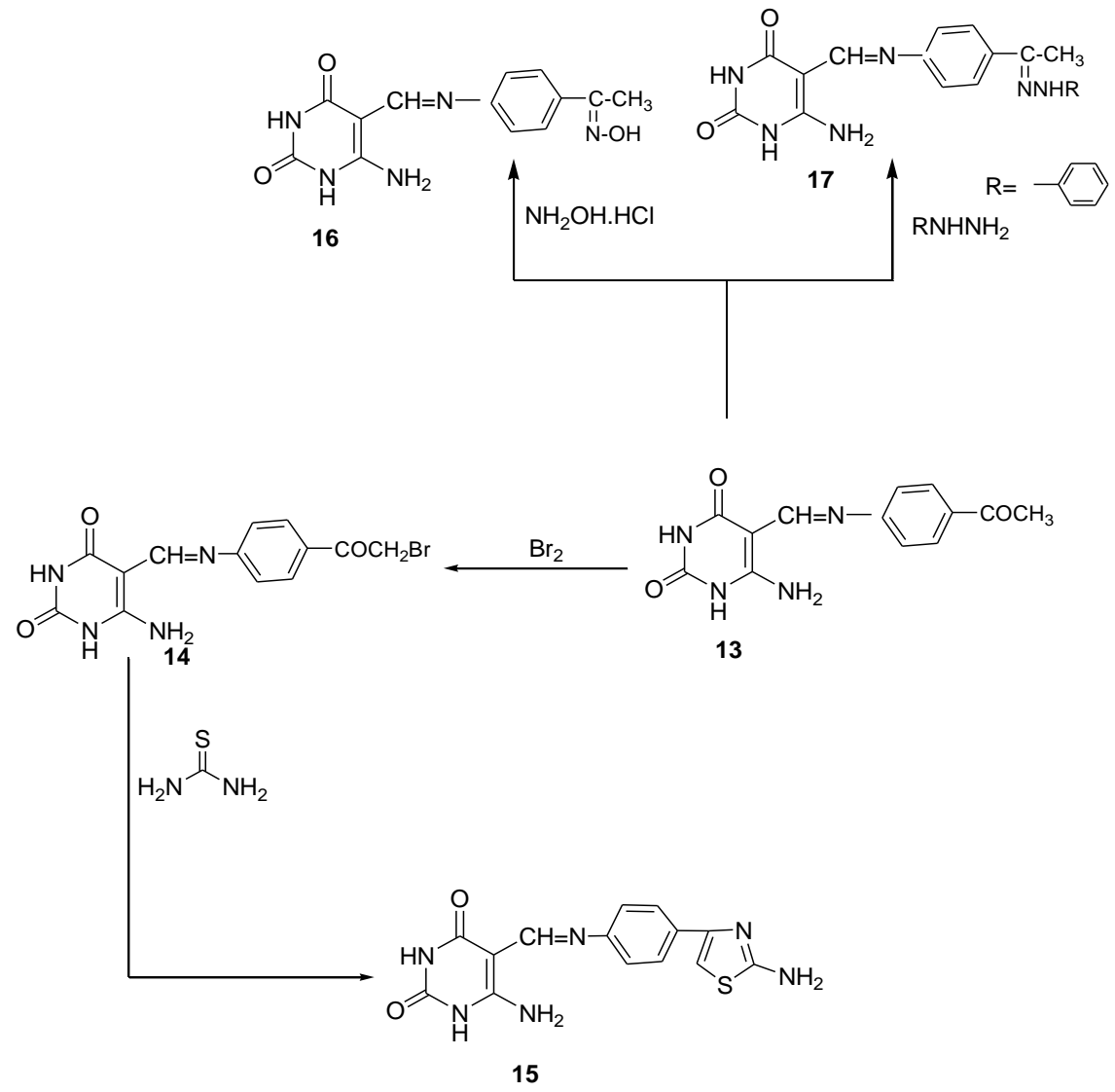

Scheme 3

\section{Experimental}

All melting points are uncorrected and were determined in capillary tube on open capillaries on Gallenkamp apparatus. IR spectra were recorded on a Beckman infrared spectrophotometer PU9712 using $\mathrm{KBr}$ discs. The $\mathrm{H}^{1}$-NMR spectra were obtained on Joel EX270, 500 MHZ spectrometer using TMS as internal standard. Mass spectra were recorded on Finngan SSQ7000 mass spectrometer at 70 e.v. All reactions were followed and checked by T.L.C using chloroform / methanol (9:3) and spots were examined by UV lamp. The compounds throughout this work were named according to the IUPAC system using Chem. Draw Ultra computer program version 8. 
1H-pyrrolo[2,3-d]pyrimidine-2,4,5,6(3H,7H)-tetrone (2)

A solution of oxalyl chloride $(0.122 \mathrm{~mol}, 17 \mathrm{ml})$ in acetone $(100 \mathrm{ml})$, was added drop wise to a suspension of compound 1 (15.5 g, $0.122 \mathrm{~mol})$ in acetone and dimethyl formamide $(10 \mathrm{ml})$. The reaction mixture was refluxed for $4 \mathrm{hr}$, the excess solvent was removed under reduced pressure and the cooled yellow precipitated material 2 was collected by filtration, washed with acetone and ethanol, dried and recrystallized from $\mathrm{DMF} / \mathrm{H}_{2} \mathrm{O}$ 1:1 to give compound 2 (Tables $1 \& 2$ ).

5-[(4-Acetylphenyl)imino]-5,7-dihydro-1H-pyrrolo[2,3-d] pyrimidine-2,4,6(3H)trione (3)

To a solution of compound $2(0.181 \mathrm{~g}, 0.001 \mathrm{~mol})$ in glacial acetic acid $5 \mathrm{ml}$, was added p-aminoacetophenone $(0.135 \mathrm{~g}, 0.001 \mathrm{~mol})$. The reaction mixture was refluxed for $20 \mathrm{hr}$, the excess solvent was removed under reduced pressure and the solid collected by filtration, washed with water and ether, dried and recrystallized from $\mathrm{DMF} / \mathrm{H}_{2} \mathrm{O}$ 1:1 to give compound 3 (Tables 1 \& 2).

$N$ - pyridin-2-yl or pyrimidin-2-yl or isoxazol-2-yl-4-\{[(5Z)-2,4,6-trioxo-1,2,3, 4,6,7hexahydro-5H-pyrrolo[2,3-d]pyrimidin-5-ylidene] amino\} benzenesul fonamides $(4 a-c)$

To a solution of compound $2(0.181 \mathrm{~g}, 0.001 \mathrm{~mol})$ in glacial acetic acid $(5 \mathrm{ml})$ was added sulfa drugs namely: sulfapyridine, sulfapyrimidine and sulfaisoxazole $(0.001 \mathrm{~mol})$. The reaction mixture was refluxed for $7-20 \mathrm{hr}$, the excess solvent was removed under reduced pressure and the solid collected by filtration, washed with water and ether, dried and recrystallized from $D M F / H_{2} \mathrm{O} \quad 1: 1$ to give compounds (4a-c) (Tables $1 \& 2$ ).

1H-pyrrolo[2,3-d]pyrimidine-2,4,5,6(3H,7H)-tetrone 5 thiosemicarbazone (5)

To a solution of compound $2(0.181 \mathrm{~g}, 0.001 \mathrm{~mol})$ in absolute ethanol $5 \mathrm{ml}$ and glacial acetic acid $(5 \mathrm{ml})$, was added thiosemicarbazide $(0.001 \mathrm{~mol})$. The reaction mixture was refluxed for $20 \mathrm{hr}$, the excess solvent was removed under reduced pressure and the solid collected by filtration, washed with water and ether, dried and recrystallized from $\mathrm{DMF} / \mathrm{H}_{2} \mathrm{O} 1: 1$ to give compound 5 (Tables $1 \& 2$ ).

Ethyl(2,4,5,6-tetraoxo-1,2,3,4,5,6-hexahydro-7H-pyrrolo [2,3-d] pyrimidin-7-yl) acetate (6)

A mixture of compound $2(0.181 \mathrm{~g}, 0.001 \mathrm{~mol})$, ethyl chloroacetate $(0.001 \mathrm{~mol})$ and sodium carbonate anhydrous $(0.083 \mathrm{~g}, 0.001 \mathrm{~mol})$ in dimethyl-formamide $(5 \mathrm{ml})$ was stirred at room temperature for $1 \mathrm{hr}$, then refluxed for $10 \mathrm{hr}$. The reaction mixture was concentrated, cold and the solid formed was filtered, washed with ethanol and ether then recrystallized from DMF to give compound 6 (Tables $1 \& 2$ ).

(2)-3-Oxo-3-phenyl-2-(2,4,6-trioxo-1,2,3,4,6,7-hexahydro-5H-pyrrolo [2,3-d] pyrimidin5 - ylidene) propanamide (7)

To a solution of compound $2(0.181 \mathrm{~g}, 0.001 \mathrm{~mol})$ in absolute ethanol $(5 \mathrm{ml})$ and glacial acetic acid $(5 \mathrm{ml})$, was added phenacylcyanide $(0.145 \mathrm{~g}, 0.001 \mathrm{~mol})$. The reaction mixture was refluxed for $14 \mathrm{hr}$, the excess solvent was removed under reduced pressure and the solid collected by filtration, washed with water and ether, dried and recrystallized from $\mathrm{DMF} / \mathrm{H}_{2} \mathrm{O} 1: 1$ to give compound 7 (Tables 1 \& 2).

Egypt. J. Chem. 54, No.5 (2011) 
(5)-5-(\{4-[(2)-3-(3,4,5- Trimethoxyphenyl ) prop-2- enoyl] phenyl\} imino)-5,7dihydro-1H-pyrrolo[2,3-d]pyrimidine-2,4,6(3H)-trione (8)

A mixture of compound $3(0.298 \mathrm{~g}, 0.001 \mathrm{~mol})$ and 3,4,5-trimethoxybenzaldehyde $(0.196 \mathrm{~g}, 0.001 \mathrm{~mol})$ in absolute ethanol $(5 \mathrm{ml})$ and $\mathrm{KOH}(10 \%, 5 \mathrm{ml})$ was stirred at room temperature for $48 \mathrm{hr}$ then refluxed for $1 \mathrm{hr}$. The reaction mixture was concentrated, cooled and poured into ice cold water. The solid formed after neutralization with dil. $\mathrm{HCl}$ was filtered off, washed with water and recrystallized from $\mathrm{DMF} / \mathrm{H}_{2} \mathrm{O}$ 1:1 to give compound 8 (Tables 1 \& 2).

2-Oxo-4-(3,4,5-trimethoxyphenyl)-6-(4-\{[(5Z)-2,4,6-trioxo-1,2,3,4,6,7-hexahydro5H-pyrrolo [2,3-d] pyrimidin-5-ylidene] aminolphenyl)-1,2-dihydropyridine-3carbonitrile (9)

A mixture of chalcone derivative $8(0.476 \mathrm{~g}, 0.001 \mathrm{~mol})$, ethyl cynoacetate $(0.001 \mathrm{~mol})$ and ammonium acetate $(0.006 \mathrm{~mol})$ in ethanol $(25 \mathrm{ml})$ was refluxed for $10 \mathrm{hr}$. The reactions mixtures were concentrated, the excess solvent was removed under reduced pressure, cooled and the solid formed was filtered off, washed with water for several times, dried then recrystallized from $\mathrm{DMF} / \mathrm{H}_{2} \mathrm{O}$ $1: 1$ to give compound 9 (Tables $1 \& 2$ ).

2-Imino-4-(3,4,5-trimethoxyphenyl-6-(4-\{[(5z)-2,4,6-trioxo-1,2,3,4,6,7-hexahydro5H-pyrrolo [2,3-d] pyrimidin-5-ylidene] amino phenyl)-1,2-dihydropyridine-3carbonitrile (10)

A mixture of chalcone derivative $8(0.476 \mathrm{~g}, 0.001 \mathrm{~mol})$, malononitrile $(0.001$ $\mathrm{mol})$ and ammonium acetate $(0.006 \mathrm{~mol})$ in ethanol $(25 \mathrm{ml})$ was refluxed for 16 $\mathrm{hr}$, the excess solvent was removed under reduced pressure, cooled and the solid formed was filtered off, washed with water for several times, dried then recrystallized from $\mathrm{DMF} / \mathrm{H}_{2} \mathrm{O}$ 1:1 to give compound 10 (Tables 1 \& 2).

(5Z)-5- \{[4- (6-(3,4,5- trimethoxyphenyl)-2- thioxo-1,2- dihydropyrimidin-4-yl) phenyl]imino 3-5,7-dihydro-1H-pyrrolo[2,3-d]pyrimidine-2,4,6(3H)-trione (11)

A mixture of chalcone derivative $8(0.476 \mathrm{~g}, 0.001 \mathrm{~mol})$, thiourea, $(0.001 \mathrm{~mol})$ and a few drops of hydrochloric acid in ethanol $(25 \mathrm{ml})$ was refluxed for $30 \mathrm{hr}$. The reaction mixture was concentrated, the excess solvent was removed under reduced pressure, cooled and the solid formed was filtered, washed with ethanol and ether then recrystallized from $\mathrm{DMF} / \mathrm{H}_{2} \mathrm{O}$ 1:1 to give compound 11 (Tables 1 \& 2).

2-Oxo-4-(indolyl)-6-(4-\{[(5)-2,4,6-trioxo-1,2,3,4,6,7-hexahydro-5H-pyrrolo[2,3-d] pyrimidin-5-ylidene Jamino phenyl)-1,2-dihydropyridine-3-carbonitrile (12)

A mixture of acetyl derivative $3(0.298 \mathrm{~g}, 0.001 \mathrm{~mol})$, ethyl cynoacetate $(0.001$ $\mathrm{mol})$, 3-indolcarboxaldehyde $(0.001 \mathrm{~mol})$ and ammonium acetate $(0.006 \mathrm{~mol})$ in ethanol $(25 \mathrm{ml})$ was refluxed for $10 \mathrm{hr}$, the excess solvent was removed under reduced pressure, cooled and the solid formed was filtered, washed with water for several times, dried then recrystallized from DMF to give compound 12 (Tables $1 \& 2$ ). 
TABLE 1. The physical and analytical data of the newly synthesized compounds (2-17).

\begin{tabular}{|c|c|c|c|c|c|c|}
\hline \multirow{2}{*}{$\begin{array}{l}\text { Comp. } \\
\text { No. }\end{array}$} & \multirow{2}{*}{$\begin{array}{l}\text { M.P. } \\
{ }^{0} \mathrm{C}\end{array}$} & \multirow{2}{*}{ Yield \% } & \multirow{2}{*}{$\begin{array}{c}\text { Mol. Formula } \\
\text { (Mol.wt.) }\end{array}$} & \multicolumn{3}{|c|}{$\begin{array}{c}\text { Analysis \% } \\
\text { Calcd./ Found }\end{array}$} \\
\hline & & & & C & $\mathbf{H}$ & $\mathbf{N}$ \\
\hline 2 & $>330$ & 81 & $\begin{array}{c}\mathrm{C}_{6} \mathrm{H}_{3} \mathrm{~N}_{3} \mathrm{O}_{4} \\
(181.17)\end{array}$ & $\begin{array}{l}39.79 \\
39.94\end{array}$ & $\begin{array}{l}1.76 \\
1.77\end{array}$ & $\begin{array}{l}23.20 \\
23.29\end{array}$ \\
\hline 3 & $>330$ & 75 & $\begin{array}{c}\mathrm{C}_{14} \mathrm{H}_{10} \mathrm{~N}_{4} \mathrm{O}_{4} \\
(298.254)\end{array}$ & $\begin{array}{l}56.38 \\
56.62\end{array}$ & $\begin{array}{l}3.38 \\
3.39\end{array}$ & $\begin{array}{l}18.78 \\
18.85\end{array}$ \\
\hline $4 a$ & $>300$ & 70 & $\begin{array}{c}\mathrm{C}_{17} \mathrm{H}_{12} \mathrm{~N}_{6} \mathrm{O}_{5} \mathrm{~S} \\
(412.3)\end{array}$ & $\begin{array}{l}49.51 \\
49.31\end{array}$ & $\begin{array}{l}2.93 \\
2.92\end{array}$ & $\begin{array}{l}20.38 \\
20.29\end{array}$ \\
\hline $4 b$ & $>300$ & 75 & $\begin{array}{c}\mathrm{C}_{16} \mathrm{H}_{11} \mathrm{~N}_{7} \mathrm{O}_{5} \mathrm{~S} \\
(413.3)\end{array}$ & $\begin{array}{l}46.49 \\
46.57\end{array}$ & $\begin{array}{l}2.68 \\
2.69\end{array}$ & $\begin{array}{l}23.72 \\
23.66\end{array}$ \\
\hline $4 c$ & $>300$ & 61 & $\begin{array}{c}\mathrm{C}_{15} \mathrm{H}_{10} \mathrm{~N}_{6} \mathrm{O}_{6} \mathrm{~S} \\
(402.3)\end{array}$ & $\begin{array}{l}44.78 \\
44.81\end{array}$ & $\begin{array}{l}2.51 \\
2.52\end{array}$ & $\begin{array}{l}20.89 \\
20.84\end{array}$ \\
\hline 5 & $>330$ & 60 & $\begin{array}{c}\mathrm{C}_{7} \mathrm{H}_{6} \mathrm{~N}_{6} \mathrm{O}_{3} \mathrm{~S} \\
(254.2)\end{array}$ & $\begin{array}{l}33.07 \\
33.20\end{array}$ & $\begin{array}{l}2.38 \\
2.37\end{array}$ & $\begin{array}{l}33.06 \\
33.19\end{array}$ \\
\hline 6 & $>330$ & 60 & $\begin{array}{c}\mathrm{C}_{10} \mathrm{H}_{9} \mathrm{~N}_{3} \mathrm{O}_{6} \\
(267.1)\end{array}$ & $\begin{array}{l}44.95 \\
44.87\end{array}$ & $\begin{array}{l}3.40 \\
3.38\end{array}$ & $\begin{array}{l}15.73 \\
15.77\end{array}$ \\
\hline 7 & $>330$ & 62 & $\begin{array}{c}\mathrm{C}_{15} \mathrm{H}_{10} \mathrm{~N}_{4} \mathrm{O}_{5} \\
(326.2)\end{array}$ & $\begin{array}{l}55.22 \\
55.40\end{array}$ & $\begin{array}{l}3.09 \\
3.10\end{array}$ & $\begin{array}{l}17.17 \\
17.10\end{array}$ \\
\hline 8 & $>300$ & 60 & $\begin{array}{c}\mathrm{C}_{24} \mathrm{H}_{20} \mathrm{~N}_{4} \mathrm{O}_{7} \\
(476.4)\end{array}$ & $\begin{array}{l}60.50 \\
60.64\end{array}$ & $\begin{array}{l}4.23 \\
4.21\end{array}$ & $\begin{array}{l}11.76 \\
11.71\end{array}$ \\
\hline 9 & $>330$ & 61 & $\begin{array}{c}\mathrm{C}_{27} \mathrm{H}_{20} \mathrm{~N}_{6} \mathrm{O}_{7} \\
(540.4)\end{array}$ & $\begin{array}{l}60.03 \\
60.16 \\
\end{array}$ & $\begin{array}{l}3.73 \\
3.71 \\
\end{array}$ & $\begin{array}{l}15.55 \\
15.49 \\
\end{array}$ \\
\hline 10 & $>330$ & 62 & $\begin{array}{c}\mathrm{C}_{27} \mathrm{H}_{21} \mathrm{~N}_{7} \mathrm{O}_{6} \\
(539.4)\end{array}$ & $\begin{array}{l}60.11 \\
60.17\end{array}$ & $\begin{array}{l}3.92 \\
3.93\end{array}$ & $\begin{array}{l}18.17 \\
18.20\end{array}$ \\
\hline 11 & $>330$ & 56 & $\begin{array}{c}\mathrm{C}_{25} \mathrm{H}_{22} \mathrm{~N}_{6} \mathrm{O}_{6} \mathrm{~S} \\
(534.3)\end{array}$ & $\begin{array}{l}56.17 \\
56.25\end{array}$ & $\begin{array}{l}4.15 \\
4.16\end{array}$ & $\begin{array}{l}15.72 \\
15.78\end{array}$ \\
\hline 12 & $>330$ & 53 & $\begin{array}{c}\mathrm{C}_{26} \mathrm{H}_{15} \mathrm{~N}_{7} \mathrm{O}_{4} \\
(489.2)\end{array}$ & $\begin{array}{l}63.80 \\
63.68 \\
\end{array}$ & $\begin{array}{l}3.09 \\
3.10 \\
\end{array}$ & $\begin{array}{l}20.01 \\
20.09 \\
\end{array}$ \\
\hline 14 & $>330$ & 64 & $\begin{array}{c}\mathrm{C}_{13} \mathrm{H}_{11} \mathrm{Br} \mathrm{N}_{4} \mathrm{O}_{3} \\
(351.1)\end{array}$ & $\begin{array}{l}44.46 \\
44.28\end{array}$ & $\begin{array}{l}3.16 \\
3.14\end{array}$ & $\begin{array}{l}15.96 \\
15.91\end{array}$ \\
\hline 15 & $>330$ & 71 & $\begin{array}{c}\mathrm{C}_{14} \mathrm{H}_{12} \mathrm{~N}_{6} \mathrm{O}_{2} \mathrm{~S} \\
(328.350)\end{array}$ & $\begin{array}{l}51.21 \\
51.41\end{array}$ & $\begin{array}{l}3.68 \\
3.66 \\
\end{array}$ & $\begin{array}{l}25.59 \\
25.69\end{array}$ \\
\hline 16 & $>330$ & 83 & $\begin{array}{c}\mathrm{C}_{13} \mathrm{H}_{13} \mathrm{~N}_{5} \mathrm{O}_{3} \\
(287.2)\end{array}$ & $\begin{array}{l}54.35 \\
54.50\end{array}$ & $\begin{array}{l}4.56 \\
4.54\end{array}$ & $\begin{array}{l}24.38 \\
24.28\end{array}$ \\
\hline 17 & $>330$ & 94 & $\begin{array}{c}\mathrm{C}_{19} \mathrm{H}_{18} \mathrm{~N}_{6} \mathrm{O}_{2} \\
\quad(362.3)\end{array}$ & $\begin{array}{l}62.97 \\
62.79\end{array}$ & $\begin{array}{l}5.01 \\
5.03\end{array}$ & $\begin{array}{l}23.19 \\
23.28\end{array}$ \\
\hline
\end{tabular}

Egypt. J. Chem. 54, No.5 (2011) 
TABLE 2. Spectral data of the newly synthesized compounds (2-17).

\begin{tabular}{|c|c|c|c|}
\hline $\begin{array}{c}\text { Comp. } \\
\text { No. }\end{array}$ & IR $\left(\mathrm{KBr}, \mathrm{cm}^{-1}\right)$ & $\begin{array}{c}{ }^{1} \mathrm{H}-\mathrm{NMR}, \underset{\text { ppm })}{\left(\mathrm{DMSO}-\mathrm{d}_{6}, \delta\right.} \\
\delta\end{array}$ & Mass $\mathbf{m} / \mathbf{z}(\%)$ \\
\hline 2 & $\begin{array}{l}3409.49,3177.96 \\
(3 \mathrm{NH}), 1685.73,(4 \mathrm{CO}), \\
1616.86(\mathrm{C}=\mathrm{N}, \mathrm{C}=\mathrm{C})\end{array}$ & $\begin{array}{l}11.20,11.65(2 \mathrm{~s}, 3 \mathrm{H}, 3 \mathrm{NH}, \\
\left.\text { exchangeable with } \mathrm{D}_{2} \mathrm{O}\right)\end{array}$ & $\begin{array}{l}\text { MS: }[\mathrm{M}+1]^{+} 182.37 \\
(13.22),[\mathrm{M}-1]^{+} 180.67 \\
(16.48),[\mathrm{M}-2]^{+} 179.66 \\
(4.10), 152.15(100), \\
77.19(64.55) .\end{array}$ \\
\hline 3 & $\begin{array}{l}3148.93(3 \mathrm{NH}), \\
1750.00,1674.00, \\
1649.75(4 \mathrm{CO}), 1595.72 \\
(\mathrm{C}=\mathrm{N})\end{array}$ & $\begin{array}{l}2.10\left(\mathrm{~s} .3 \mathrm{H}, \mathrm{CH}_{3}\right), 7.70,7.90 \\
(\mathrm{dd}, 4 \mathrm{H}, \mathrm{Ar}-\mathrm{H}), 8.50,10.25, \\
10.50(3 \mathrm{~s}, 3 \mathrm{H}, 3 \mathrm{NH}, \\
\left.\text { exchangeable with } \mathrm{D}_{2} \mathrm{O}\right) .\end{array}$ & $\begin{array}{l}\text { MS: } \text { M }^{+} 298(10), 247 \\
(20), 203(100), 78(83), \\
62(95) .\end{array}$ \\
\hline $4 a$ & $\begin{array}{l}\text { 3288.04, 3141.47 (NH), } \\
2992.98(\mathrm{CH}, \text { aliphatic), } \\
1702.84(3 \mathrm{CO}), 1610.27 \\
(\mathrm{C}=\mathrm{C}, \mathrm{C}=\mathrm{N}) .\end{array}$ & $\begin{array}{l}\text { 6.80-8.10 (m, 8H, Ar-H), } \\
10.30(\mathrm{~s}, 3 \mathrm{H}, 3 \mathrm{NH}), 11.80 \\
\text { (br. s., } 1 \mathrm{H}, \mathrm{SO}_{2} \mathrm{NH}, \\
\left.\text { exchangeable with } \mathrm{D}_{2} \mathrm{O}\right)\end{array}$ & $\begin{array}{l}\text { MS: } \mathrm{M}^{+} 412(0.16),[\mathrm{M}- \\
1]^{+} 411(0.15), 374 \\
(42.51), 373(100), 256 \\
(3.19), 247(5.26), 233 \\
(3.85), 179(2.12) .\end{array}$ \\
\hline $4 b$ & $\begin{array}{l}\text { 3423.03, 3354.57, } \\
\text { 3256.22 (NH), } \\
\text { 3036.37(CH aromatic), } \\
\text { 1716.34, 1651.73 (3CO), } \\
1585.20(\mathrm{C}=\mathrm{C}, \mathrm{C}=\mathrm{N}), \\
1322.93,1145.19\left(\mathrm{SO}_{2}\right) .\end{array}$ & $\begin{array}{l}6.10(\mathrm{~s}, \mathrm{H}, \mathrm{NH}, \\
\left.\text { exchangeable with } \mathrm{D}_{2} \mathrm{O}\right), \\
6.55-8.50(\mathrm{~m}, 7 \mathrm{H}, \mathrm{Ar}-\mathrm{H}) \\
11.30,11.60,12.00(3 \mathrm{~s}, 3 \mathrm{H}, \\
\text { 3NH, exchangeable with } \\
\left.\mathrm{D}_{2} \mathrm{O}\right) .\end{array}$ & $\begin{array}{l}\text { MS: } \mathrm{M}^{+} 413(5), 380(3), \\
322(5), 247(65), 78(80), \\
63(100) .\end{array}$ \\
\hline $4 c$ & $\begin{array}{l}3176.19(\mathrm{NH}), 3038.30 \\
(\mathrm{CH} \text { aromatic }) 1690.30 \\
(3 \mathrm{CO}), 1617.98(\mathrm{C}=\mathrm{C}, \\
\mathrm{C}=\mathrm{N}), 1310.00,1160.94 \\
\left(\mathrm{SO}_{2}\right) .\end{array}$ & $\begin{array}{l}\text { 7.60-9.10 }(\mathrm{m}, 6 \mathrm{H}, \mathrm{Ar}-\mathrm{H}), \\
10.40(\mathrm{~s}, 1 \mathrm{H}, \mathrm{NH}, \\
\left.\text { exchangeable with } \mathrm{D}_{2} \mathrm{O}\right) \\
11.60,12.00(2 \mathrm{~s}, 3 \mathrm{H}, 3 \mathrm{NH} \text {, } \\
\left.\text { exchangeable with } \mathrm{D}_{2} \mathrm{O}\right)\end{array}$ & $\begin{array}{l}\text { MS: }[\mathrm{M}+1]^{++} 403(2), 327 \\
(3), 247(25), 110(5), 93 \\
(100) .\end{array}$ \\
\hline 5 & $\begin{array}{l}3410.49,3174.26, \\
3039.26\left(\mathrm{NH}, \mathrm{NH}_{2}\right), \\
1703.80,(3 \mathrm{CO}), 1617.98 \\
(\mathrm{C}=\mathrm{C}, \mathrm{C}=\mathrm{N}), 1079.94 \\
(\mathrm{C}=\mathrm{S}) .\end{array}$ & $\begin{array}{l}8.50\left(\mathrm{~s}, 2 \mathrm{H}, \mathrm{NH}_{2}\right), 10.21, \\
10.50(2 \mathrm{~s}, 2 \mathrm{H}, 2 \mathrm{NH}, \\
\left.\text { exchangeable with } \mathrm{D}_{2} \mathrm{O}\right) \\
11.59-12.00(2 \mathrm{~s}, 2 \mathrm{H}, 2 \mathrm{NH} \text { of } \\
\text { pyrimidine ring, } \\
\left.\text { exchangeable with } \mathrm{D}_{2} \mathrm{O}\right) .\end{array}$ & $\begin{array}{l}\text { MS: }[\mathrm{M}+2]^{++} 256(18.23), \\
{[\mathrm{M}+1]^{+} 255(0.68), 192} \\
(4.87), 64(100) .\end{array}$ \\
\hline 6 & $\begin{array}{l}3182.93,(\mathrm{NH}), 3055.66 \\
(\mathrm{CH}, \text { aromatic }), 2928.38 \\
(\mathrm{CH} \text { Aliph), 1723.09, } \\
1677.77(5 \mathrm{CO}), 1604.48 \\
(\mathrm{C}=\mathrm{C}, \mathrm{C}=\mathrm{N}) .\end{array}$ & $\begin{array}{l}1.20\left(\mathrm{t}, 3 \mathrm{H}, \mathrm{CH}_{2}-\mathrm{CH}_{3}\right), 4.10 \\
\left(\mathrm{q}, 2 \mathrm{H}, \mathrm{CH}_{2}-\mathrm{CH}_{3}\right), 4.70,5.00 \\
\left(\mathrm{dd}, 2 \mathrm{H}, \mathrm{NCH}_{2} \mathrm{CO}\right), 8.50, \\
8.80(2 \mathrm{~s}, 2 \mathrm{H}, 2 \mathrm{NH}, \\
\left.\text { exchangeable with } \mathrm{D}_{2} \mathrm{O}\right) .\end{array}$ & $\begin{array}{l}\text { MS: } M^{+} 267(5), 250(9), \\
237(10), 193(7), 174 \\
(100), 181(13), 167(63), \\
149(95) .\end{array}$ \\
\hline 7 & $\begin{array}{l}3179.0,\left(\mathrm{NH}, \mathrm{NH}_{2}\right), \\
3032.00(\mathrm{CH}, \text { aromatic }), \\
1715.49(5 \mathrm{CO}) .\end{array}$ & $\begin{array}{l}4.40\left(\mathrm{~s}, 2 \mathrm{H}, \mathrm{NH}_{2},\right. \\
\left.\text { exchangeable with } \mathrm{D}_{2} \mathrm{O}\right), \\
6.40-8.90(\mathrm{~m}, 5 \mathrm{H}, \mathrm{Ar}-\mathrm{H}), \\
9.00(\mathrm{~s}, \mathrm{H}, \mathrm{NH} \text { of pyrrole } \\
\text { ring, exchangeable with } \\
\left.\mathrm{D}_{2} \mathrm{O}\right), 11.50 \text { (br. s, } 2 \mathrm{H}, 2 \mathrm{NH} \text {, } \\
\left.\text { exchangeable with } \mathrm{D}_{2} \mathrm{O}\right) .\end{array}$ & $\begin{array}{l}\text { MS: } \mathrm{M}^{+} 326(2.91), \\
247.01(83.91), 205 \\
(7.80), 178(3.2), 177 \\
(11), 174(100) .\end{array}$ \\
\hline
\end{tabular}




\begin{tabular}{|c|c|c|c|}
\hline \multicolumn{4}{|c|}{ TABLE 2. Cont. } \\
\hline $\begin{array}{l}\text { Comp. } \\
\text { No. }\end{array}$ & $\operatorname{IR}\left(\mathrm{KBr}, \mathbf{c m}^{-1}\right)$ & $\begin{array}{c}{ }^{1} \mathrm{H}-\mathrm{NMR},\left(\mathrm{DMSO}-\mathrm{d}_{6}, \delta\right. \\
\text { ppm) }\end{array}$ & Mass m/z (\%) \\
\hline 8 & $\begin{array}{l}3181.97(\mathrm{NH}), 3011.30 \\
(\mathrm{CH}, \text { aromatic), } 2816.53 \\
\text { (CH aliph.), 1722.12, } \\
1678.73(4 \mathrm{CO}), 1602.56 \\
(\mathrm{C}=\mathrm{C}, \mathrm{C}=\mathrm{N}) .\end{array}$ & $\begin{array}{l}3.70\left(\mathrm{~s}, 3 \mathrm{H}, \mathrm{p}-\mathrm{OCH}_{3}\right), 3.85 \\
\left(\mathrm{~s}, 6 \mathrm{H}, 2 \mathrm{~m}-\mathrm{OCH} \mathrm{CH}_{3}\right), 7.20-9.40 \\
(\mathrm{~m}, 8 \mathrm{H}, \mathrm{Ar}-\mathrm{H}, \mathrm{CH}=\mathrm{CH},) \\
11.50,12.00,(2 \mathrm{~s}, 3 \mathrm{H}, 3 \mathrm{NH}, \\
\left.\text { exchangeable with } \mathrm{D}_{2} \mathrm{O}\right)\end{array}$ & $\begin{array}{l}\text { MS: }[\mathrm{M}+1]^{+} 477.46 \\
(0.08), 212(100), 197 \\
(49.22), 168.18(0.63), \\
169(10.98) .\end{array}$ \\
\hline 9 & $\begin{array}{l}\text { 3325.64, 3177.15, }(\mathrm{NH}) \\
\text { 3046.98, }(\mathrm{CH}, \\
\text { Aromatic) } 2210.99 \\
(\mathrm{C} \equiv \mathrm{N}), 1695.12(4 \mathrm{CO}), \\
1617.02(\mathrm{C}=\mathrm{C}, \mathrm{C}=\mathrm{N}) .\end{array}$ & $\begin{array}{l}3.80\left(\mathrm{~s}, 3 \mathrm{H}, \mathrm{p}-\mathrm{OCH}_{3}\right), 4.00(\mathrm{~s}, \\
\left.6 \mathrm{H}, 2 \mathrm{~m}-\mathrm{OCCH})_{3}\right), 6.65-9.00 \\
(\mathrm{~m}, 7 \mathrm{H}, \mathrm{Ar}-\mathrm{H}, \mathrm{CH} \text { of } \\
\text { pyridone ring), } 9.32,11.11 \\
\text { (s, br. s 4H, 4NH). }\end{array}$ & $\begin{array}{l}\text { MS: } \mathrm{M}^{+} 514(10), 324 \\
(25), 247(100), 204(85), \\
177(65), 78(55) .\end{array}$ \\
\hline 10 & $\begin{array}{l}3414.35,3170.40,(\mathrm{NH}, \\
\left.\mathrm{NH}_{2}\right), 3038.30(\mathrm{CH}, \\
\text { aromatic) } 2225(\mathrm{C} \equiv \mathrm{N}), \\
1685.48(3 \mathrm{CO}), 1615.09 \\
(\mathrm{C}=\mathrm{C}, \mathrm{C}=\mathrm{N})\end{array}$ & $\begin{array}{l}3.70\left(\mathrm{~s}, 3 \mathrm{H}, \mathrm{p}-\mathrm{OCH}_{3}\right), 3.80 \\
\left(\mathrm{~s}, 6 \mathrm{H}, 2 \mathrm{~m}-\mathrm{OCH}_{3}\right), 8.00-9.40 \\
(\mathrm{~m}, 7 \mathrm{H}, \mathrm{Ar}-\mathrm{H}, \mathrm{CH} \text { of } \\
\text { iminopyrdine ring), } 11.50 \\
\text { (br. s, 5H, 5NH, } \\
\left.\text { exchangeable with } \mathrm{D}_{2} \mathrm{O}\right) \text {. }\end{array}$ & $\begin{array}{l}\text { MS: } \mathrm{M}^{+} 539(0.53), \\
372.26(5.3), 373.51 \\
(18.76), 174(100) .\end{array}$ \\
\hline 11 & $\begin{array}{l}3426.89(\mathrm{NH}), 2817.49 \\
(\mathrm{CH} \text { Aliph.), } 1690.70 \\
(3 \mathrm{CO}), 1598.70 \quad(\mathrm{C}=\mathrm{C}, \\
\mathrm{C}=\mathrm{N}), 1129.12(\mathrm{C}=\mathrm{S}) .\end{array}$ & & $\begin{array}{l}\text { MS: } \mathrm{M}^{+} 534(2.19), \\
443.21(35.61), 441.28 \\
(50.76), 355.05(33.29), \\
356.17(40.48), 174.20 \\
(100), 167.25(11.43) .\end{array}$ \\
\hline 12 & $\begin{array}{l}3172.85,(\mathrm{NH}), 3064.84 \\
(\mathrm{CH}, \text { aromatic }), 2212.18 \\
(\mathrm{C} \equiv \mathrm{N}), 1694.18(4 \mathrm{CO}), \\
1619.20(\mathrm{C}=\mathrm{N}) .\end{array}$ & $\begin{array}{l}7.25-8.60(\mathrm{~m}, 10 \mathrm{H}, \mathrm{Ar}-\mathrm{H}, \\
\mathrm{CH} \text { of pyridone ring), } 8.90 \\
(\mathrm{~s}, \mathrm{H}, \mathrm{NH} \text { of pyridone ring), } \\
9.00 \mathrm{~s}, \mathrm{H}, \mathrm{NH} \text { of indole } \\
\text { ring), } 9.35 \text { (s, H, NH of } \\
\text { pyrrole ring), } 11.80 \text { (br. s. } \\
2 \mathrm{H}, 2 \mathrm{NH}) .\end{array}$ & $\begin{array}{l}\text { MS: } \mathrm{M}^{+} 489(7), 472(30), \\
446(50), 389(100), 390 \\
(30), 373(10), 317(55) .\end{array}$ \\
\hline 14 & $\begin{array}{l}3350,3200 \quad\left(\mathrm{NH}, \mathrm{NH}_{2}\right), \\
1750,1650.70 \quad(3 \mathrm{CO}), \\
1600(\mathrm{C}=\mathrm{N})\end{array}$ & $\begin{array}{l}4.95\left(\mathrm{~s}, 2 \mathrm{H}, \mathrm{CH}_{2} \mathrm{Br}\right), 6.60(\mathrm{~s}, \\
2 \mathrm{H}, \mathrm{NH}_{2} \text {, exchangeable with } \\
\left.\mathrm{D}_{2} \mathrm{O}\right), 7.60-8.20(\mathrm{~m}, 4 \mathrm{H}, \mathrm{Ar}- \\
\mathrm{H}), \quad 8.60 \quad(\mathrm{t}, \quad \mathrm{H}, \quad \mathrm{CH}=\mathrm{N}) \\
10.95,11.20(2 \mathrm{~s}, 2 \mathrm{H}, 2 \mathrm{NH}, \\
\left.\text { exchangeable with } \mathrm{D}_{2} \mathrm{O}\right) .\end{array}$ & $\begin{array}{l}\text { MS: } \mathrm{M}^{+} 351(6), 247(25), \\
155(85), 127(100) .\end{array}$ \\
\hline 15 & $\begin{array}{l}3250,3180.74(\mathrm{NH}, \\
\left.\mathrm{NH}_{2}\right), 1750,1720(2 \mathrm{CO}), \\
1625.00(\mathrm{C}=\mathrm{N})\end{array}$ & $\begin{array}{l}6.2\left(\mathrm{~s}, 2 \mathrm{H}, \mathrm{NH}_{2} \text { of pyrimidine }\right. \\
\text { ring, exchangeable with } \\
\left.\mathrm{D}_{2} \mathrm{O}\right), 7.15\left(\mathrm{~s}, 2 \mathrm{H}, \mathrm{NH}_{2} \text { of }\right. \\
\text { thiazole ring exchangeable } \\
\left.\text { with } \mathrm{D}_{2} \mathrm{O}\right), 7.60-8.10(\mathrm{~m}, 5 \mathrm{H} \text {, } \\
\mathrm{Ar}-\mathrm{H} \text { and } \mathrm{CH} \text { of thiazole } \\
\text { ring), }), 8.70,(\mathrm{t}, \mathrm{H}, \mathrm{CH}=\mathrm{N}) \text {, } \\
10.95,11.10(2 \mathrm{~s}, 2 \mathrm{H}, 2 \mathrm{NH}, \\
\left.\text { exchangeable with } \mathrm{D}_{2} \mathrm{O}\right) \text {. }\end{array}$ & $\begin{array}{l}\text { MS: } \mathrm{M}^{+} 328(5), 256(30), \\
160(20), 128(35), 63 \\
(100) .\end{array}$ \\
\hline
\end{tabular}

Egypt. J. Chem. 54, No.5 (2011) 


\begin{tabular}{|c|c|c|c|}
\hline \multicolumn{4}{|c|}{ TABLE 2. Cont. } \\
\hline $\begin{array}{l}\text { Comp. } \\
\text { No. }\end{array}$ & IR $\left(\mathrm{KBr}, \mathrm{cm}^{-1}\right)$ & $\begin{array}{c}{ }^{1} \mathrm{H}-\mathrm{NMR},\left(\mathrm{DMSO}-\mathrm{d}_{6}, \delta\right. \\
\text { ppm) }\end{array}$ & Mass m/z (\%) \\
\hline 16 & $\begin{array}{l}3402.78,3345.89, \\
3202.22\left(\mathrm{NH}, \mathrm{NH}_{2},\right. \\
\mathrm{OH}), 2997.80(\mathrm{CH}, \\
\text { aliph.), 1729.83, (2CO), } \\
\text { 1632.45 (C=C, C=N). }\end{array}$ & $\begin{array}{l}2.5\left(\mathrm{~s}, 3 \mathrm{H}, \mathrm{CH}_{3}\right), 7.40,8.10 \\
(\mathrm{dd}, 4 \mathrm{H}, \mathrm{Ar}-\mathrm{H}), 9.30(\mathrm{~s}, 2 \mathrm{H}, \\
\mathrm{NH}_{2}, \text { exchangeable with } \\
\left.\mathrm{D}_{2} \mathrm{O}\right), 9.70(\mathrm{~s}, \mathrm{H}, \mathrm{CH}=\mathrm{N}) \\
10.40,10.70,10.80(3 \mathrm{~s}, 3 \mathrm{H}, \\
2 \mathrm{NH}, \mathrm{OH}, \text { exchangeable } \\
\left.\text { with } \mathrm{D}_{2} \mathrm{O}\right) .\end{array}$ & \\
\hline 17 & $\begin{array}{l}3410.49,3175.22(\mathrm{NH}, \\
\left.\mathrm{NH}_{2}\right), 3039.26(\mathrm{CH}, \\
\text { aromatic), } 1704.76 \\
(2 \mathrm{CO}), 1617.98(\mathrm{C}=\mathrm{C}, \\
\mathrm{C}=\mathrm{N}) .\end{array}$ & $\begin{array}{l}2.50\left(\mathrm{~s}, 3 \mathrm{H}, \mathrm{CH}_{3}\right), 6.50-7.30 \\
(\mathrm{~m}, 9 \mathrm{H}, \mathrm{Ar}-\mathrm{H}), 8.10(\mathrm{~s}, \mathrm{H}, \\
\mathrm{CH}=\mathrm{N}), 8.40(\mathrm{br} . \mathrm{s}, 2 \mathrm{H}, \\
\left.\mathrm{NH}_{2}\right), 9.75(\mathrm{~s}, 1 \mathrm{H}, \mathrm{NH}), \\
10.60(\mathrm{~s}, 2 \mathrm{H}, 2 \mathrm{NH} \text { of } \\
\text { pyrimidine ring, } \\
\left.\text { exchangeable with } \mathrm{D}_{2} \mathrm{O}\right) .\end{array}$ & $\begin{array}{l}\text { MS: }[\mathrm{M}+1]^{+} 363.51 \\
(4.12), 285(3.55), 174 \\
(100), 128(48.39), 126.17 \\
(4.11) .\end{array}$ \\
\hline
\end{tabular}

6-Amino-5- (-\{[4-(bromoacetyl) phenyl] imino\} methyl) pyrimidine-2,4(1H,3H)dione (14)

A mixture of the acetyl derivative ${ }^{(14)} 13(0.273 \mathrm{~g}, 0.001 \mathrm{~mol})$, bromine $(0.001$ mol), glacial acetic acid $(15 \mathrm{ml})$ was stirred for $48 \mathrm{hr}$, the excess solvent was removed under reduced pressure, cooled and the solid formed was filtered, washed with water for several times, dried then recrystallized from DMF to give compound 14 (Tables 1 \& 2).

6-Amino-5-(-\{[4-(2-amino-1,3-thiazol-4-yl) phenyl]imino $\}$ methyl) pyrimidine-2,4 $(1 \mathrm{H}, 3 \mathrm{H})$-dione $(15)$

A mixture of bromoacetyl derivative $14(0.001 \mathrm{~mol})$, thiourea, $(0.001 \mathrm{~mol})$ in $(25$ $\mathrm{ml}$ ) ethanol was refluxed for $7 \mathrm{hr}$, the excess solvent was removed under reduced pressure, cooled and the solid formed was filtered, washed with ethanol and ether then recrystallized from $\mathrm{DMF} / \mathrm{H}_{2} \mathrm{O}$ 1:1 to give compound 15 (Tables 1 \& 2).

6-Amino-5-[-(\{4-[(1)-N-hydroxyethanimidoyl]phenyl $\}$ imino $)$ methyl] pyrimidine$2,4(1 H, 3 H)$-dione (16)

To a solution of compound $13^{(14)}(0.273 \mathrm{~g}, 0.001 \mathrm{~mol})$ in absolute ethanol $(5 \mathrm{ml})$, was added hydroxylamine hydrochloride $(0.001 \mathrm{~mol})$. The reaction mixture was refluxed for $7 \mathrm{hr}$, the excess solvent was removed under reduced pressure and the solid collected by filtration, washed with water and ether, dried and recrystallized from $\mathrm{DMF} / \mathrm{H}_{2} \mathrm{O}$ 1:1 to give compound 16 (Tables $1 \& 2$ ).

6-Amino-5-[-(\{4-[(1)-N-phenylethanehydrazonoyl]phenyl $\}$ imino $)$ methyl Jpyrimidine2,4(1H,3H)-dione (17)

To a solution of compound ${ }^{(14)} 13(0.273 \mathrm{~g}, 0.001 \mathrm{~mol})$ in absolute ethanol (5 $\mathrm{ml})$ and glacial acetic acid $(5 \mathrm{ml})$, was added phenylhydrazine $(0.001 \mathrm{~mol})$. The reaction mixture was refluxed for $8 \mathrm{hr}$, the excess solvent was removed under reduced pressure and the solid collected by filtration, washed with water and ether, dried and recrystallized from $\mathrm{DMF} / \mathrm{H}_{2} \mathrm{O}$ 1:1 to give compound 17 (Tables $1 \& 2$ ). 
Antimicrobial activity of the referred chemical compounds using agar-diffusion method

For the provided compounds (coded as $4 \mathrm{a}, 4 \mathrm{~b}, 4 \mathrm{c}, 5,6,8,9,12,15$ and 16), antimicrobial activity was studied using agar-diffusion method.

\section{Materials and Methods}

Organisms

All microbial strains used were local isolates and obtained from National Research Center, Cairo, Egypt. Antibacterial activity was tested against Escherichia coli (Gram negative short rods), Staphylococcus aureus (Gram positive cocci) and Bacillus subtilis (Gram positive spore-forming bacilli). Antifungal activity was tested against Aspergillus niger (mould) and Candida albicans (yeast). Inocula of $24 \mathrm{hr}$ age from each strain (except in case of Aspergillus niger, $72 \mathrm{hr}$ age inoculum has been used) were prepared and used in seeding bioassay media.

\section{Media}

Antimicrobial activity was assayed in agar plates of medium 1 (for testing antibacterial activity) or medium 2 (for testing antifungal activity). Molten sterile $40 \mathrm{ml}$ of medium were allowed to cool to $45^{\circ} \mathrm{C}$ before seeding with the test strain and poured in Petri dish of $15 \mathrm{~cm}$. diameter.

$\begin{array}{ll}\text { Medium 1 } & \\ \text { Peptone } & 5 \mathrm{gm} \\ \text { Glucose } & 5 \mathrm{gm} \\ \text { Beef extract } & 3 \mathrm{gm} \\ \text { Yeast extract } & 1 \mathrm{gm} \\ \text { Agar } & 11 \mathrm{gm} \\ \text { Distilled water } & 1 \mathrm{~L} \\ \text { pH 7 } & \end{array}$

$\begin{array}{ll}\text { Medium 2 } & \\ \text { Peptone } & 2 \mathrm{gm} \\ \text { Glucose } & 5 \mathrm{gm} \\ \text { Agar } & 11 \mathrm{gm} \\ \text { Distilled water } & 1 \mathrm{~L} \\ \mathrm{pH} \mathrm{7} & \end{array}$

\section{Results}

Each studied compound was loaded on filter paper disc (Whattmann No. 3) of $6.5 \mathrm{~mm}$ diameter and allowed to dry in air. Discs loaded with tested compounds were gently overlaid on the surface of agar media under sterile conditions. Then, the agar plates with discs were maintained in refrigerator for $30 \mathrm{~min}$ before incubation for $48 \mathrm{hr}$ (in case of Aspergillus niger) or $24 \mathrm{hr}$ (for all other strains). Inhibition zone less than $6.5 \mathrm{~mm}$ refers to inability of studied compound to exert any inhibition of microbial growth under studied conditions. 
TABLE 3. Antimicrobial activity of the provided compounds using agar- diffusion method.

\begin{tabular}{|c|c|c|c|c|c|c|c|c|c|c|c|c|}
\hline & \multicolumn{7}{|c|}{ Antimicrobial activity of the studied compounds* } \\
\cline { 5 - 13 } Test & $\begin{array}{c}\text { Reference } \\
\text { ontimi- } \\
\text { crobianism } \\
\text { agent }\end{array}$ & $\begin{array}{c}\text { Reference } \\
\text { antimi- } \\
\text { crobial } \\
\text { agent }\end{array}$ & $\mathbf{4 a}$ & $\mathbf{4 b}$ & $\mathbf{4 c}$ & $\mathbf{5}$ & $\mathbf{6}$ & $\mathbf{8}$ & $\mathbf{9}$ & $\mathbf{1 2}$ & $\mathbf{1 5}$ & $\mathbf{1 6}$ \\
\hline $\begin{array}{c}\text { Escherichia } \\
\text { coli }\end{array}$ & $\begin{array}{c}\text { Fusidine } \\
10 \mu \mathrm{g}\end{array}$ & 18 & $<6.5$ & $<6.5$ & $<6.5$ & $<6.5$ & $<6.5$ & 7 & 7 & $<6.5$ & 12 & 8 \\
\hline $\begin{array}{c}\text { Staphylococ } \\
\text { cus aureus }\end{array}$ & $\begin{array}{c}\text { Fusidine } \\
10 \mu \mathrm{g}\end{array}$ & 17 & 10 & $<6.5$ & $<6.5$ & $<6.5$ & $<6.5$ & $<6.5$ & 7 & $<6.5$ & 8.5 & $<6.5$ \\
\hline $\begin{array}{c}\text { Bacillus } \\
\text { subtilis }\end{array}$ & $\begin{array}{c}\text { Fusidine } \\
10 \mu \mathrm{g}\end{array}$ & 26 & $<6.5$ & $<6.5$ & $<6.5$ & $<6.5$ & $<6.5$ & $<6.5$ & $<6.5$ & $<6.5$ & 11 & $<6.5$ \\
\hline $\begin{array}{c}\text { Candida } \\
\text { albicans }\end{array}$ & $\begin{array}{c}\text { Rapamycin } \\
0.3 \mu \mathrm{g}\end{array}$ & 18 & $<6.5$ & $<6.5$ & $<6.5$ & $<6.5$ & $<6.5$ & $<6.5$ & $<6.5$ & $<6.5$ & $<6.5$ & $<6.5$ \\
\hline $\begin{array}{c}\text { Aspergillus } \\
\text { niger }\end{array}$ & $\begin{array}{c}\text { Rapamycin } \\
0.3 \mu \mathrm{g}\end{array}$ & 13 & $<6.5$ & $<6.5$ & $<6.5$ & $<6.5$ & $<6.5$ & $<6.5$ & $<6.5$ & $<6.5$ & $<6.5$ & $<6.5$ \\
\hline
\end{tabular}

* Expressed as the diameter of inhibition zone in $\mathrm{mm}$.

\section{Conclusion for Antimicrobial Activity}

From Table 3 all of the tested compounds showed no antimicrobial activity except compound $4 \mathrm{a}$ which showed slight activity against staphylococcus aureus, this may be due to the presence of pyridine sulfonamide in para-position of phenyl ring while compound 15 showed slight activity against Escherichia coli and Bacillus subtilis, this may be due to the presence of amino thiazole group in the para-position of phenyl ring.

\section{References}

1. Ingarsal, N., Saravanan, G., Amutha, P. and Nagarajan, S., Eur. J. Med. Chem. xx, 1 (2006).

2. Davarani, S.S.H., Fumani, N.S. and Moradi, H.A.N.F., Tetrahedron Lett. 49, 710 (2008).

3. Ali, A., Aster, S.D., Graham, D.W., Patel, G.F., Taylor, G.E., Tolman, R.L., Painter, R.E., Silver, L.L., Young, E.K., Geissler, W. and Harris, G.S., Bioorg. Med. Chem. Lett. 11, 2185 (2001).

4. Wahid, F., Monneret, C. and Dauzonne, D., Chem. Pharm. Bull. 47 (2), 156 (1999).

5. Fellahi, Y., Dubois, P., Mandin, D., Goka, J.E.O., Guenzet, J., Chaumont, J.P. and Frangin, Y., Eur. J. Med. Chem. 30, 633 (1995).

6. Tumkevicius, S. and Sarakauskaite, Z., Polish J. Chem. 27, 1275 (2003).

7. Cobo, J., Sanchez, A. and Nogueras, M., Tetrahedron , 54, 5753 (1998).

8. Ballell, L., Field, R.A., Chung, G.A.C. and Young, R.J., Bioorg. Med. Chem. Lett. 17, 1736 (2007). 
9. Bernier, J.L. and Henichart, J.P., J. Heter. Chem. 16, 717 (1979).

10. Abdel-Rahman, A.H.F., Journal of the Chinese Chemical Society, 15, 147 (2004).

11. El-Emary, T.I., Ahmad, R.A. and Bakhite, E.A., Journal of the Chinese Chemical Society, 48, 921 (2001).

12. Makhlouf, A.A., Kamel, M.M., Anwar, M.M., Haiba, M.E. and Mohei El-Deen, E.M., Egypt. Pharm. J. 4 (2), 371 (2005).

13. Fathalla, O.A., Awad, S.M. and Mohamed, M.S., Arch. Pharm. Res. 28 (11), 1205 (2005).

14. Nofal, Z.M., Fahmy, H.H., Zarie, E.S. and El-Eraky, W., Acta Poloniae Pharmaceutica-Drug Research, 68 (4), 507 (2011).

15. Fahmy, H.H., Egypt J. Pharm. Sci. 38, 403 (1997).

(Received 3/ 8/2011; accepted 13/10/2011)

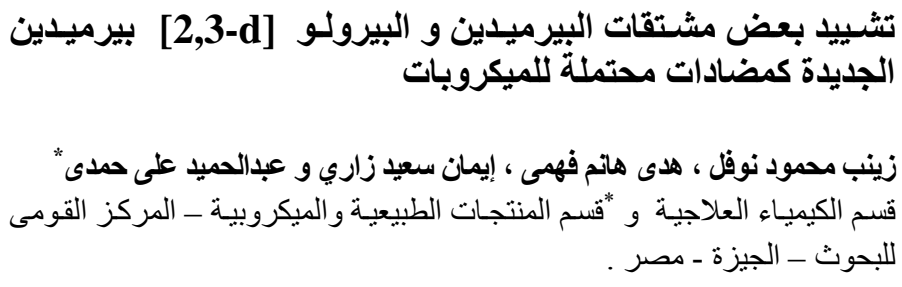

Egypt. J. Chem. 54, No.5 (2011) 